\title{
Index of birds
}

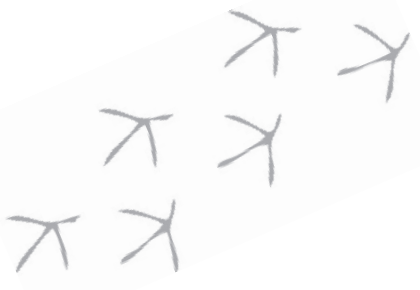

Common names follow the BOU British List (Harrop et al., 2013) for species recorded in Britain and the IOC World Birds List, Master IOC list v5.4 for other species. Where these differ the IOC name is in parentheses. See appendices 1-3. Page numbers in italic refer to illustrations; 'n.' after a page number indicates the number of a note on that page.

Auk, Great 65, 157, 227, 239, 272

Avocet, American 39

Babax, Giant 222, 223

Bee-eater

Arabian Green 173, plate 34

Blue-bearded plate 59

Blue-tailed 175

Böhm's 171

Red-bearded plates 35 and 59

Bittern

American 24, 86

(Eurasian) Bittern 81, 270

Blackbird (Common Blackbird) 13, 155, 256

Brush Finch

Bay-crowned 181

White-winged 181

Bufflehead 24, 93

Bullfinch

Azores 107-8

Cinereous 183n.12

Bunting

Pine 138, 145

Rustic 145, 278

Snow 87

Bustard, Great 182, 271

Buzzard

Common 213

Rough-legged 252

Chachalaca, Plain 38

Chaffinch (Common Chaffinch) 172
Chough (Red-billed Chough) 153-4, 253

Cormorant, Pygmy 210

Crane

Hooded 238-9

Siberian 125, 226, 271

Whooping 42

Crossbill (Red Crossbill) 253-4, plate 22

Cuckoo (Common Cuckoo) 74, 79, 254, 273

Curlew

Eskimo 73, 112, 264

Slender-billed 134, 226-7, 263-4, plate 61

Dipper (White-throated Dipper) 186, 207, 208, 277

Diver, Great Northern (Great Northern Loon) 24, 269

Dotterel (Eurasian Dotterel) 151n.8

Dowitcher

Asian 226, plate 56

Short-billed 39

Duck

Harlequin 93, 146, 268

Labrador 93

Dunlin plate 29

Eagle

Bald 24, 29

Bonelli's 107

Booted 57, 73

Golden 82

Imperial 109

Indian Spotted 162 
Lesser Spotted 162

Spanish Imperial 182

Spotted (Greater Spotted Eagle) 162, plate 31

Tawny 139, 162, 194, 206

Eagle-Owl

Eurasian 25, 146, 148, 273

Siberian 214

Egret

Great White (Great Egret) 146

Snowy 39, 45

Eider

American 144-5

Common 112, plate 62

King plate 62

Spectacled 226, plate 55

Steller's plates 2, 60 and 62

Falcon; see also Peregrine

Gyr (Gyrfalcon) 87, 94, 114, 274, plate 1; see also 'Labrador Falcon'

'Labrador' 114, 154, 198, 210, plates 1, 19 and 47

Red-footed 138

Saker 109, plate 28

Flamingo, Greater 182, 269

Fulmar (Northern Fulmar) 194

Goosander (Common Merganser) 116n.12 Goose

Lesser Snow 80

Snow 42

Grebe

Great Crested 255

Slavonian (Horned Grebe) 103, 269

Greenshank 178, plate 38

Grosbeak, Pine 25, 278

Ground Roller, Short-legged plate 36

Grouse

Black 26, 79

Black $\times$ Hazel (hybrid) 189

Black $\times$ Red (hybrid) 80

Caucasian 110, plate 18

Hazel 94

Red 211, 268

Ruffed 24, 94

Spruce 93-4

Gull

Audouin's 107

Ivory 115

Ross's 113, 208, 224-7, 263, plates 52-4
Heron, Tricolored 45

Ibis

American White 39

Glossy 57 , plate 7

Northern Bald 108-9, plate 17

Sacred 69, 70, 214-15

Ibisbill 214, 226

Kestrel

American 145

Lesser 145

Kingfisher

Belted 86

Common 81, 273

Kite

Red 251, 252

Swallow-tailed 89-90, 93

Knot (Red Knot) 104, 114, 208, 212-13, 219-20, plate 16

Lark

'Eastern Shore' ('Eastern Horned Lark') 177 , plate 37

Shore (Horned Lark) 29, 177

'Steppe Shore' ('Steppe Horned Lark') 144

White-winged 145, 275

Laughingthrush, Brown-cheeked 223

Magpie

Azure-winged 57, 93, 274

(Eurasian) Magpie 208

Martin, Purple 86

Merganser, Hooded 71-2, 89, plate 14

Nightingale (Common Nightingale) 57, 239

Nuthatch

Eastern Rock 213

Krüper's, 109

Osprey (Western Osprey) 94, 187, 270

Owl; see also Eagle-Owl

Barn (Western Barn Owl) 81

Snowy 29, 115, plate 20

Tengmalm's (Boreal Owl) 140

Oystercatcher

African Black 183n.15

Canary Islands 172, plate 44

Partridge, Daurian 187

Pelican, Brown 45 
Index of birds

Peregrine (Peregrine Falcon) 94, 253, plate 58

Petrel

Fea's (Desertas Petrel) 186, plate 45

Leach's 89, 93, 251, 269

Soft-plumaged plate 45

Phalarope

Grey (Red Phalarope) 151n.8

Red-necked 151n.8, 271

Pheasant

Common 236

Copper 211

Pigeon

Bolle's 107-8, 272

Passenger 24

Pipit, Pechora 144, 250, plate 57

Plover

Grey 104, 106-7

Pacific Golden 80-1

Rufous-chested 196

White-tailed (White-tailed Lapwing) 133

Prairie Chicken, Greater 40

Pratincole, Collared 182

Ptarmigan, Willow 112, 140; see also Grouse, Red

Puffin (Atlantic Puffin) 194

Quail, Montezuma 42

Raven (Northern Raven), 'Pied' 105

Redshank 255

Redwing 73, 214, 277

Roadrunner, Greater 38

Robin

American 24

(European) Robin 57, 256

Roller; see also Ground Roller

Cuckoo 173

European 138

Purple 185, plate 43

Racket-tailed 185-6, plate 42

Rook 221

Sanderling 104, 114, 212

Sandgrouse, Pallas's 85, 260-1, 272

Sandpiper

Buff-breasted 40, plate 4

Curlew 57, 212, plate 6

'Eastern Broad-billed' 144
Green 252

Marsh 138

Pectoral plate 54

Spoon-billed 208

Spotted 146

Upland 40

Scoter, Surf 93, plate 15

Serin, Syrian 181, plate 40

Shrike

Great Grey 153, 204-5, 274, plate 48

Grey-backed 223

Isabelline 250

Red-backed 239, 253, 267n.7

Woodchat 138

Skimmer, Black 39

Skua, Great 105, 236

Smew 25, 105, plate 3

Snipe (Common Snipe) 116n.18

Snowcock, Caspian 108, 144

Sparrow

Dead Sea 214

House 78, 221, 277

Spoonbill, Roseate 39

Starling, Common 26, 213, 276

Stilt, Black-necked 39

Stint

Little 57, 104, 106-7, 115, plate 29

Temminck's plate 29

Stonechat, Canary Islands 172

Stork, Black 54, 94, 139, 270

Swallow (Barn Swallow) 145, 275

Teal

Baikal 138

Eurasian 138

Tern

Arctic 144, plate 24

Black 39, 57

Common 144

Sooty 93

Thrush, Grey-backed 194, 207

Tit

African Blue 172, 275

'British Coal' 145, plate 25

'British Marsh' 181, plate 41

Coal 145, plate 25

Crested 138

Long-tailed 145, 186, 191, 207, 276

Marsh 181, plate 41

Turkey, Wild 41 


\section{Wagtail}

'Grey-headed' (subspecies of Yellow Wagtail) 248n.13, 277

Yellow 277, plate 21

Warbler

Garden 239, 276

Golden-cheeked 73, 278, plate 13

Hume's (Hume's Leaf Warbler) 167n.30, plate 30

Moustached 250-1

Pallas's 187 , plate 46

River 251

Rüppell's 250

Yellow-browed $167 \mathrm{n} .30$, plate 30
Waxwing

Bohemian 20-1, 24-5, 73, 82, 145, 197, 275 , plate 12

Cedar 93, 145, 187

Wheatear

Black-eared 144, 178, 181, plate 39

Seebohm's 204-6, plate 49

Wigeon, American 80

Woodpecker

American Three-toed 86

Black 145, 154, 274, plate 26

Ivory-billed 73

'Lilford's' 145, 181

Wren (Common Wren), 178-9, 276

St Kilda 178, 181, 207 